\title{
Endometrial evaluation with methylene blue staining in patients with normal hysteroscopy
}

\author{
Alka Goel, Pooja Gupta*, Akansha Singh, Khushboo Singh
}

Department of Obstetrics and Gynecology, PGIMER and Dr. RML Hospital, New Delhi, India

Received: 27 April 2019

Accepted: 30 May 2019

*Correspondence:

Dr. Pooja Gupta,

E-mail: drpoojagupta1 @gmail.com

Copyright: () the author(s), publisher and licensee Medip Academy. This is an open-access article distributed under the terms of the Creative Commons Attribution Non-Commercial License, which permits unrestricted non-commercial use, distribution, and reproduction in any medium, provided the original work is properly cited.

\begin{abstract}
Background: Normally, endometrium comprises of non-absorptive epithelium and does not take up stain. Conventional staining with methylene blue is explained on the basis of existence of apoptotic cells in endometrium. Methods: Of 50 patients of unexplained infertility, AUB, recurrent pregnancy loss were randomly selected and included in the study. Those with abnormal ultrasound and history of tuberculosis were excluded. Conventional hysteroscopy was performed using normal saline as distending medium and in those with grossly normal endometrium were subjected to staining with 5\% methylene blue instilled trans cervically. After 5 min, irrespective of the size and pattern, focal dark blue stained areas were considered abnormal and randomly biopsied. Incidence of endometritis in both groups was compared after histopathological examination.

Results: Of total 50 patients, histopathological report of only one patient with dark blue staining had evidence of endometritis. Rest had no evidence, of which $73.5 \%$ had light blue or unstained areas and $26.5 \%$ showed dark blue staining. No statistically significant difference was found between histopathological reports and light or dark blue staining $(\mathrm{p}=0.28)$. When percentage stained area was considered more than $50 \%$ only to be positive, sensitivity was $100 \%$, specificity $94 \%$, PPV $25 \%$ and NPV 100\%. False positives were $75 \%$ and no false negatives were observed. Although $\mathrm{p}$ values improved but still statistically insignificant.

Conclusions: Present study failed to establish any significant correlation between staining pattern and detection rate of endometritis. With no Indian studies published on chromohysteroscopy so far, role of methylene blue in detection of subtle endometrial changes in modern gynaecology in Indian subpopulation is yet to be established.
\end{abstract}

Keywords: Chromohysteroscopy, Endometritis, Histopathology, Methylene blue, Staining

\section{INTRODUCTION}

Hysteroscopy today is considered as a minimally invasive process to diagnose and treat various endometrial and endocervical pathologies through transcervical approach. ${ }^{1}$ Uterine cavity receptivity and integrity are among the major factors affecting various physiological functions. Hence, when no obvious factor is found for abnormal uterine bleeding, infertility and early pregnancy failure, then endometrium warrants evaluation. ${ }^{2,3}$ Although uterine evaluation has been done traditionally through ultrasound, sonosalpingography and hysteronsalpingography.

Hysteroscopy allows direct visualization of uterine cavity and provides opportunities for simultaneous treatment of various pathologies. In cases when no apparent abnormality is detected on hysteroscopy and the uterus is considered normal, it may not actually be so and the cells may still be abnormal. Hysteroscopy has limited value in the detection of subtle endometrial changes at the cellular level which fail to produce macroscopic abnormality. 
Endometrial staining during conventional hysteroscopy with vital stains (1\% methylene blue) can be used to detect apoptotic endometrial cells. Under normal circumstances, endometrium is a non-absorptive epithelium and does not take up any dye. ${ }^{4}$ The American Society for Reproductive Medicine emphasized that the cells and their membranes must be damaged to allow methylene blue dye to reach cytoplasm and stain the nucleus. ${ }^{5}$

Minimal apoptosis is always present in endometrium due to cyclical shedding during menstruation. Hence endometrium gives light blue stain, which is considered normal. Whereas, dark blue staining represents marked apoptosis due to endometritis. Targeted endometrial biopsies can be taken to diagnose cellular findings by histopathological examination.

The present study was designed to study the role of methylene blue staining in endometrial evaluation in patients with normal hysteroscopy, find an association if any between the staining pattern and detection rates of endometritis, and whether chromohysteroscopy can be of value as a technique in improving the diagnostic yield in evaluation of endometrium.

\section{METHODS}

A total of 50 patients were recruited during a period of 18 months (September 2012- February 2014) from among those attending the gynaecology outpatient department of PGIMER and Dr. RML Hospital. They were found to have no apparent reason, for abnormal uterine bleeding, early pregnancy failure or infertility and were thus indicated for hysteroscopy. The patients were recruited according to the following inclusion criteria.

\section{Inclusion criteria}

The patients with apparently normal hysteroscopy with any of the following:

- Patients with abnormal uterine bleeding

- Patients with 2 or more spontaneous abortions during the first trimester due to unexplained reasons with last abortion more than 3 months prior to inclusion

- Patients of unexplained infertility for more than 1year cohabiting with husband.

\section{Exclusion criteria}

- Patients with obvious gross pathology on hysteroscopy which included-fibroids, polyps, congenital anomalies of uterus, endometrial hyperplasia and endometrial malignancy

- Patients with a current and past history of treated for tuberculosis

- Patients diagnosed to have tubercular endometritis through prior endometrial biopsy

- Patients with diagnosed bleeding diathesis.
Each patient short-listed for the study was subjected to routine investigation including pelvic ultrasound. Those with normal findings were then selected for hysteroscopy. A conventional hysteroscopy was done using $4 \mathrm{~mm} 30$ degree Foreoblique rigid hysteroscope. Normal saline was used as the distension medium.

Hysteroscope was gently manipulated into uterine cavity under vision. Panoramic view was followed by a systematic examination of all four walls of uterine cavity and tubal openings. Patients in whom no gross abnormality was detected in the uterine cavity, endometrial staining was performed using methylene blue.

After conventional hysteroscopy, distending medium flow was stopped and hysteroscope was removed. Foleys catheter was inserted intracervically and 5-ml of $1 \%$ methylene blue was introduced through it. After 5 minutes, foleys catheter was removed. Hysteroscope was introduced again and distension media was restarted to wash the endometrium. Uterine cavity was visualized for the amount and pattern of staining. Diffuse light blue staining was considered normal and a hysteroscopic guided biopsy was taken. Focal dark blue staining was considered abnormal and targeted biopsies were taken using hysteroscopic grasping forceps.

Endometritis by histopathological examination in patients with dark staining areas and light staining areas were then compared. For histopathological examination, tissue samples taken were paraffin processed. Sections were cut at 4-microns and stained with hematoxylin and eosin stains.

\section{Statistical analysis}

Statistical analysis was done with the SPSS software. Continuous variables are presented as mean \pm SD. Categorical variables are expressed as frequencies and percentages. Nominal categorical data between groups were compared using Chi-square test and Fischer's Exact test as applicable. Standard formulae were used for calculating specificity, sensitivity, positive and negative predictive values.

\section{RESULTS}

Patients selected for study as per the inclusion and exclusion criteria, underwent conventional hysteroscopy followed by staining with methylene blue. Targeted biopsies were taken from light and dark stained areas. Comparison was done between the two histopathological reports. Results were divided tabulated according to:

- Patient characteristics: Age, BMI and parity.

- Biopsy characteristics: Light blue stained, dark blue stained.

- Endometrial area stained: Unstained, less than $50 \%$, more than $50 \%$. 
- Histopathological characteristics: Endometritis present or absent.

Of 50 patients studied, mean age was 35 years (range 21 49 years). 22 patients (44 per cent) were less than 30 years of age. 28 patients (56 per cent) had BMI between 18.5-24.9. 21 (42 per cent) of the patients were overweight and 1 was obese.

\section{Staining pattern}

Under normal circumstances, endometrium is non absorptive and does not take up any stain or stained light blue. For the endometrial cells to take up dyes, their cell membrane should be damaged as in endometritis and hence takes dark blue colour as shown in hysteroscopic image (Figure 1).

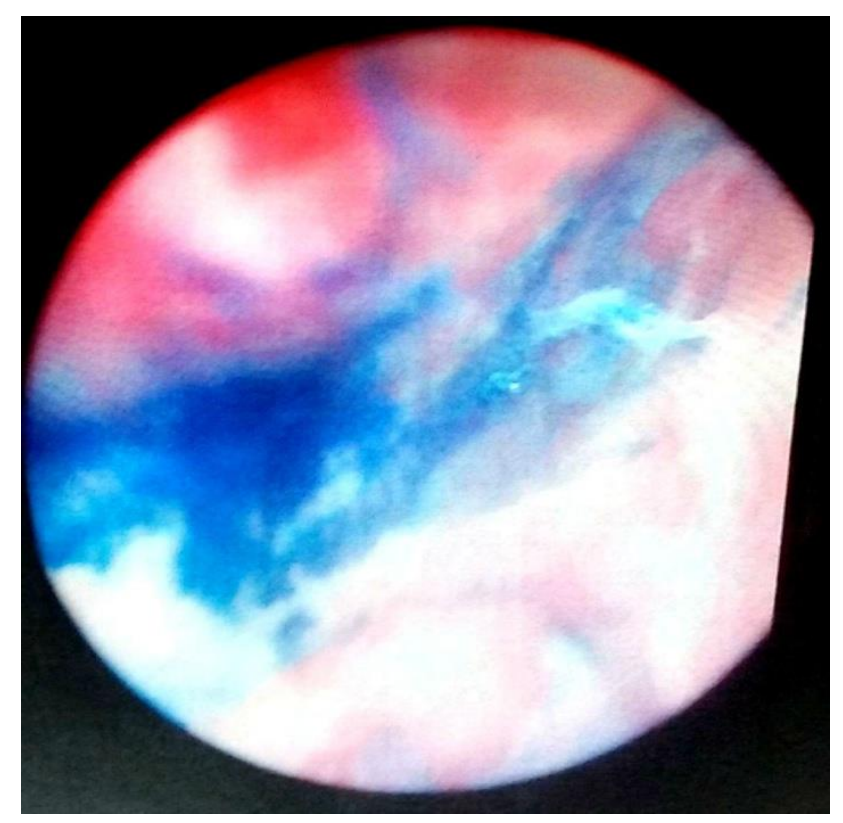

Figure 1: as-Hysteroscopic view of endometrium after staining with methylene blue.

In 30 patients (60 per cent) light blue staining was seen. In 14 patients ( 28 per cent) there was dark staining and in 6 patients (12 per cent), endometrium remained unstained.

Out of $60 \%$ patients showing light blue staining, 20 patients (two thirds or $40 \%$ of all patients) had staining in less than 50 percent of endometrial area and remaining patients (10) had staining in more than $50 \%$ of area.

Out of 14 (28 per cent) patients showing dark blue staining, in 10 patients $(20 \%$ of all) the staining was in less than $50 \%$ of endometrial area and in 4 patients (8 per cent of all), staining was present in more than $50 \%$ of endometrial area.

Out of all 50 patients, histopathology report of one patient showing dark blue staining had evidence of endometritis. Rest were reported to be normal. Of those reported to be normal, 36 patients (73.5 per cent) had light blue staining or were unstained and 13 patients $(26.5 \%)$ had dark blue staining. The difference in staining pattern when correlated with histopathology was found to be statistically insignificant $(\mathrm{p}=0.280)$.

On considering dark blue staining in more than $50 \%$ of endometrial area to be positive and rest negative, one patient with dark blue staining was detected to have endometritis on histopathology. Among patients with negative HPE report, only six percent had stained positive while rest had stained negative. This led to improved specificities and lowered false positive rates. However, this was not reflected in terms of statistical significance in the detection rates of endometritis in both groups $(\mathrm{p}=0.098)$.

\section{DISCUSSION}

Endometritis is the inflammation of endometrial lining. If chronic, it may be asymptomatic while acute cases may present as fever, abnormal bleeding, pain, discharge etc. In India, tuberculosis is a common cause of endometritis. A study conducted by Das et al, in estimated that about $18 \%$ of infertile females have been found to have tuberculosis. ${ }^{6}$ In US, however only $1 \%$ of infertile females have been found to have tuberculosis. ${ }^{7}$ Chronic endometritis is present in about $3-10 \%$ women of abnormal uterine bleeding. ${ }^{8}$

In a study conducted by Zeyneloglu et al, in observed that micro polyps in hysteroscopy is a significant predictor of miscarriage. ${ }^{9}$ But incidence of endometritis was not stated. Similar studies also have reported an association of endometritis with micro polyps. ${ }^{10-12}$

The diagnosis of endometritis is important since it can lead to infertility, abnormal bleeding and recurrent pregnancy loss. Present study aims at diagnosing endometritis at an early stage before the appearance of gross changes at endometrium. The need for such a diagnostic technique was felt which could detect subtle endometrial changes at an initial stage in an otherwise healthy looking endometrium.

Concept of staining endometrium with methylene blue was inspired from gastroenterologists who employ staining of mucosa to detect changes at cellular level. Present study aimed at discovering the role of chromohysteroscopy as a new technique to improve the diagnostic value of hysteroscopy.

\section{Patient characteristics}

No significant correlation was found between age and staining characters $(p=0.410)$. Similarly, in a study by Mohammed A et al, in who conducted chromohysteroscopy in 57 patients of recurrent abortions belonging to 20-35 years, no correlation was found 
between staining pattern and the age groups. ${ }^{13}$ Findings of the present study were similar to studies conducted by Kucuk et al, and Thornhill et al. ${ }^{14,15}$ In present study, $42 \%$ patients were overweight and $2 \%$ were obese. No significant correlation was found between BMI and staining pattern of endometrium $(\mathrm{p}=0.320)$, which is in accordance with studies by Mohammed et al, Kucuk et al and Thornhill $\mathrm{C}$ et al. ${ }^{13-15}$ Our study included 24 patients of infertility, among which $58 \%$ had primary infertility and $42 \%$ belonged to secondary infertility group. Total of 18 patients of abnormal uterine bleeding were included in the present study. Only 11 percent of the patients were nullipara, $66 \%$ multipara and $23 \%$ had one child. No significant difference was observed between the parity of patient and the staining pattern. However no previously published studies are yet available on chromohysteroscopy in patients of infertility and abnormal uterine bleeding and large scale studies are yet required to establish any correlation between staining pattern and parity.

\section{Staining characteristics}

Among a total of 50 patients recruited, $60 \%$ of the patients showed light blue staining, $28 \%$ showed dark blue staining and in $12 \%$ endometrium was unstained. Results of the present study results were similar to the results of Kucuk et al, where $28 \%$ of patients with postmenopausal bleeding showed dark blue staining. ${ }^{16} \mathrm{On}$ the contrary Kucuk et al, in found dark blue staining in $55 \%$ patients of recurrent abortions; Mohammad A et al, in found dark blue staining in $44 \%$ of patients with recurrent abortions and Thornhill et al, found dark blue staining in $38 \%$ of patients with recurrent IVF failures. ${ }^{12,14,15}$ Values of dark blue staining are higher in these studies probably explains the higher incidence of endometritis obtained in these studies.

Since light blue staining and lack of staining both were considered normal, they were clubbed together in our study for analysis. No other study has mentioned this subgroup of patients in which unstained zones were identified. Of 36 patients showing light blue or unstained areas $72.2 \%$ had staining in less than $50 \%$ of endometrial area, while in $27.7 \%$ of patients, staining was in more than $50 \%$ of the total endometrial area. However, no previous studies till now have taken the percentage of area stained into account. Histopathological reports of all light blue or unstained areas showed no evidence of endometritis. This finding was similar to those obtained in studies conducted by Kucuk et al. ${ }^{14,16}$ Negative predictive value in our study was $100 \%$. In contrast Mohammad et al, found the presence of endometritis in $7 \%$ and Thornhill et al found endometritis in $20 \%$ of patients with light blue staining of the endometrium. ${ }^{13}$

Of 14 patients showing dark blue staining, in $71.4 \%$ patients less than $50 \%$ of total endometrial area was stained, while in $28.6 \%$ patients more than $50 \%$ area was stained. In patients with staining less than $50 \%$ area, histopathological report showed normal endometrium with no evidence of endometritis. Among those with more than $50 \%$ area stained, histopathological report of one patient had evidence of tubercular endometritis.

If we consider all 14 patients which showed any amount of dark staining even in a small pocket, $7.14 \%$ showed evidence of endometritis. On the other hand, if more than $50 \%$ area is taken into consideration, then $25 \%$ ( 1 out of 4) patients showed presence of endometritis.

Sensitivity calculated for the detection of endometritis in patients with dark blue staining regardless of area was $100 \%$ and specificity was $73.5 \%$, positive predictive value $7.1 \%$ and negative predictive value was $100 \%$. This gives a false positive rate of $92 \%$. There was no statistically significant correlation between the detection rate of endometritis and staining character $(\mathrm{p}=0.28)$. These values improved substantially if percentage area stained was considered. Sensitivity improved to $94 \%$ and positive predictive value improved to $25 \%$ without compromising the sensitivity and negative predictive value which remained $100 \%$. False positive rate although reduced to $75 \%$, was still high. Moreover, no statistically significant difference was found in the detection rate of endometritis in both groups $(\mathrm{p}=0.098)$.

These findings were in contrast with other studies which showed much higher incidence of endometritis in dark stained areas (Mohammad et al in 40\%, Thornhill et al in $45.8 \%$, Kucuk et al in $52.6 \%$ in recurrent abortion group, $30 \%$ in post-menopausal group). ${ }^{13-16}$ In previous studies, focal dark blue staining suggested the presence of endometritis with sensitivity ranging $69-84 \%$ and specificity $72-95 \%$.

\section{CONCLUSION}

However present study failed to obtain a statistically significant correlation between histopathological evidence of endometritis and staining characteristics despite of taking percentage area stained into consideration. This may be due to small sample size and low incidence of endometritis in the study. This being a preliminary study to establish chromohysteroscopy as a diagnostic tool for detection of endometritis, clinical application of present study is yet to be determined.

Further studies on a larger scale with modifications are needed to refine and improve the accuracy of this technique. Broader studies with bigger sample size are required to draw definitive conclusions regarding routine use of endometrial staining during hysteroscopy.

\section{ACKNOWLEDGMENTS}

Authors would like to thank to respected teachers, family and friends for supporting me through the completion of this study. 
Funding: No funding sources

Conflict of interest: None declared

Ethical approval: The study was approved by the Institutional Ethics Committee

\section{REFERENCES}

1. Siegler, Valle, Lindemann. Diagnostic and therapeutic hysteroscopy, indications and techniques. Clin Obstetrics Gynecol. 1990;1:148-9.

2. Rowe PJ, Comhaire FH, Hargreave TB, Mellows HJ. WHO manual for the standardized investigation and diagnosis of the infertile couple. Cambridge: The Press Syndicate of the University of Cambridge. 1993:660-68.

3. Brill A. Role of hysteroscopy in management of abnormal uterine bleeding. Clin Obstetrics Gynecol. 1995;38:319-45.

4. Guillermo M, Vilela M, Quintana R, Diradourian M, Sueldo S. New observations on endometrial physiology with transcervical injection of methylene blue dye. Fertli Steril. 2004;82:1700-4.

5. Practice Committee of American Society for Reproductive Medicine in Collaborations with reproductive surgeons: Reproductive functions. Fertil Steril. 2008;90:125-30.

6. Das P, Ahuja A, Gupta SD. Incidence, Etiopathogenesis and pathological aspects of genitourinary tuberculosis in India. Ind $\mathrm{J}$ Urol. 2008;24:356-61.

7. Dey C, Watt CG, Hossieni SM. Evaluation of tuberculosis control and prospects of reducing tuberculosis incidence, prevalence and deaths globally. JAMA. 2005;293:279-93.

8. Dongen $\mathrm{H}$, Kroon $\mathrm{CD}$, Jacobi CE, Trimboss JB, Jansen FW. Diagnostic hysteroscopy in abnormal uterine bleeding: a systematic review and metanalysis. British J Obstetrics Gynecol. 2007;114:664-75.

9. Zeyneloglu A, Cicinell E, Restal, Nicoletti, Zappimbulso. Endometrial micropolyps suggest the existence of chronic endometritis. Human Reprod. 2005;20:386-9.

10. Shamma FN, Lee G, Gutmann JN, Lavy G. The role of microhysteroscopy in in-vitro fertilization. Fertil Steril. 1992:1237-9.

11. Cicenelli E, Resta L, Nicoletti R, Tartangi M, Saliani N. Endometrial micropolyps at fluid hysteroscopy suggest the existence of chronic endometritis. Human Reproductive Med. 2005;20:386-9.

12. Barash A, Dekel N, Fieldust S, Segal I, Schechtman E, Granot I. Local injury to the endometrium doubles the incidence of successful pregnancies in patients undergoing in vitro fertilization. Fertil Steril. 2003;79:1317-22.

13. Mohamed MA. Value of endometrial dyeing in diagnosis of endometritis. Med Wire. 2010:284-6.

14. Kucuk T, Safali M. Chromohysteroscopy in evaluation of endometrium in recurrent miscarriage. Eur J Gynecological Oncol. 2008:133-6.

15. Thornhill C. Chromohysteroscopy spots occult endometritis in recurrent endometritis. Eur J Gynecological Oncol. 2008:274-8.

16. Kucuk T, Devici S. Can chromohysteroscopy help target endometrial biopsy in postmenopausal women. Eur J Gynecological Oncol. 2008;29:165-7.

Cite this article as: Goel A, Gupta P, Singh A, Singh K. Endometrial evaluation with methylene blue staining in patients with normal hysteroscopy. Int J Reprod Contracept Obstet Gynecol 2019;8:2715-9. 